\title{
Information, éducation du patient et littératie en santé : un rôle à jouer pour les bibliothécaires/documentalistes en France?
}

\author{
Vincent de Andrade", Aurore Margat, Jean-Charles Verheye et Rémi Gagnayre \\ Laboratoire Éducations et Pratiques de Santé (LEPS), EA 3412, Université Paris 13 Sorbonne Paris Cité, 74 rue Marcel-Cachin, 93017 \\ Bobigny cedex, France
}

Reçu le 13 novembre 2017 / Accepté le 11 avril 2018

\begin{abstract}
Résumé - La politique de santé de plusieurs pays, dont les États-Unis, incite fortement les bibliothèques publiques à soutenir l'information et l'éducation du patient. La prise en compte de la littératie en santé des populations est un enjeu majeur. S'il existe des initiatives en France, celles-ci n'ont pas l'ampleur des expériences internationales. Pourtant, rien n'empêcherait qu'au sein des bibliothèques, les bibliothécaires/ documentalistes favorisent auprès de la population des apprentissages en santé. Une réflexion nous semble nécessaire pour concevoir, en France, l'implication des bibliothécaires/documentalistes dans ce but.
\end{abstract}

Mots clés : éducation du patient / littératie en santé / bibliothèque / bibliothécaire / documentaliste / France

\begin{abstract}
Information, patient education and health literacy: a role for librarians in France? Health policy in several countries, including the United States, strongly encourages public libraries to support patient information and education. Addressing population health literacy is a major issue. Although there are initiatives in France, they do not have the scope of international experience. Yet, nothing would prevent librarians/documentalists within libraries from promoting health literacy among the population. We feel that reflection is necessary to conceive, in France, the involvement of librarians/documentalists for this objective.
\end{abstract}

Keywords: patient education / health literacy / library / librarian / France

En mars dernier, la Bibliothèque de Médecine américaine (National Library of Medicine - NLM) et l'Institut National de Santé américain (National Institute of Health - NIH) ont co-signé une publication majeure. En effet, ces deux institutions affirment que les bibliothèques publiques (et par conséquent les bibliothécaires) peuvent répondre aux besoins d'informations en santé de la population car elles sont «d'excellentes ressources pour faire progresser la recherche et la pratique de la littératie en santé» et qu'elles sont «perçues [par le grand public] comme dignes de confiance et utile [...] en matière de santé et d'éducation du patient» [1]. Toujours aux États-Unis, dans le cadre du programme «librairies transform» de valorisation des services offerts par les bibliothèques, l'Association des Bibliothèques Américaines (ALA), la NIH et le réseau national des bibliothèques de médecine (NNLM) ont mis à disposition de leurs réseaux de bibliothèques des outils de communication (health literacy

\footnotetext{
*Auteur de correspondance :

vincent.deandrade@univ-paris13.fr
}

toolkit) [2] facilitant la compréhension des contenus des textes par les lecteurs selon leur niveau de littératie en santé. On rappellera que ce niveau situe la capacité d'un individu à savoir trouver des informations sur la santé voir médicales, les comprendre et savoir les utiliser au profit de sa santé. La littératie en santé (HL) est si influente sur l'état de santé des personnes, que l'OMS la considère comme un déterminant de la santé sur lequel des interventions doivent être envisagées lorsqu'elle est retrouvée faible chez un patient ou une population [3].

Parmi les interventions possibles qualifiées de simple ou de complexe selon l'organisation et la technicité qu'elles requièrent, l'éducation thérapeutique est considérée, encore à tort, comme inaccessible pour une personne avec un faible niveau de HL. Pourtant, moyennant l'organisation de partenariats entre le secteur sanitaire et les milieux de lutte contre l'illettrisme ainsi que des adaptations des méthodes pédagogiques, il est possible d'inverser cette situation [4].

D'autres interventions sont envisagées comme celle d'impliquer des bibliothécaires pour aider les patients à 
trouver et comprendre les informations dont ils ont besoin. Par exemple, en Italie, un centre d'oncologie a créé une bibliothèque dédiée à l'éducation des patients. Elle propose des outils d'informations co-élaborés avec les professionnels de santé (brochures) et un choix de sites Internet en santé sélectionnés pour leur fiabilité [5]. Herry et al. [6] ont montré, à partir d'une analyse de la littérature, que la présence d'un bibliothécaire au sein d'un service de santé améliore son efficacité car sa contribution permet de gagner du temps en recherches d'articles ce qui impacte significativement la qualité des soins et des coûts d'hospitalisation.

En France, il existe déjà des initiatives pour impliquer les bibliothécaires dans l'accès à des informations auprès des personnes en particulier dans le champ de l'éducation pour la santé. C'est le cas au sein de la Cité de la Santé [7,8], dans les Espaces Rencontres et Informations des centres d'oncologie notamment [9], dans les maisons des usagers [10] ou encore dans certaines structures relevant des Instituts Régionaux en Éducation pour la Santé. Les «bibliothèques d'hôpital» [11] qui n'ont pas eu le développement escompté, auraient pu jouer un rôle mais leur mode de fonctionnement reste trop aléatoire dans la mesure où il repose sur un bénévolat précaire [12]. On peut également citer les «Espaces Info Santé » présents dans plusieurs hôpitaux et qui sont des lieux de documentations et d'informations des patients. Ces espaces sont généralement animés par des professionnels de santé. À notre connaissance, il n'existe pas en France, de bibliothécaires/documentalistes impliqués actuellement dans des programmes d'ETP. Cela serait-il néanmoins possible? Le cahier des charges d'autorisation des programmes d'ETP précise que «d'autres professionnels [autres que les professionnels de santé] peuvent [y] intervenir» sans aucune autre précision [13].

Est-il besoin de rappeler qu'en France plus 15 millions de personnes atteintes d'une maladie chronique peuvent être concernées par une recherche d'informations sachant que la France se situe dans la moyenne basse de tous les pays étudiés par l'OCDE [14] concernant le niveau de littératie de sa population.

Une réflexion en terme de santé publique nous semble indispensable pour concevoir l'implication des bibliothécaires/ documentalistes dans l'information et l'éducation des patients.

Conflits d'intérêts. Les auteurs déclarent n'avoir aucun lien d'intérêt concernant les données publiées dans cet article.

\section{Références}

1. Whitney W, Keselman A, Humphreys B. Libraries and librarians: key partners for progress in health literacy research and practice. Inf Serv Use. 2017; 37(1):85-100. Disponible sur : http://content.iospress.com/articles/information-services-anduse/isu821.

2. Martin C. Libraries transform: health literacy toolkit [en ligne]. Disponible sur: https://news.nnlm.gov/pnr/libraries-transformhealth-literacy-toolkit/.
3. Kickbusch I, Pelikan MJ, Apfel F, Tsouros AD. Health literacy. The solid facts, Copenhagen: WHO regional office for Europe; 2013, vii-73 p. Disponible sur: http://www.euro.who.int/fr/ health-topics/environment-and-health/urban-health/publica tions/2013/health-literacy.-the-solid-facts.

4. Margat A, Gagnayre R, Lombrail P, de Andrade V, AzoguiLévy S. Interventions en littératie en santé et éducation thérapeutique : une revue de la littérature. Santé Publique. 2017; 29 (6):811-820.

5. Truccolo I. Providing patient information and education in practice: the role of the health librarian. Health Inf Libr J 2016; 33(2):161-166.

6. Héry L, Weill C, Macé B, Benoist D, Boutet A, Defaux H, et al. Médecins, mettez un bibliothécaire dans votre moteur (de recherche)! Pedagog Med. 2015; 16(4):251-260. Disponible sur: https://doi.org/10.1051/pmed/2016010.

7. Cité de la santé [Site Internet]. Paris: Cité des Sciences et de l'Industrie [consulté le 6 octobre 2017]. Disponible sur: http:// www.cite-sciences.fr/fr/au-programme/lieux-ressources/cite-dela-sante/.

8. Nguyên T.-T. La Cité de la Santé : un espace pour s'informer. Revue de l'Association des Bibliothécaires de France; 2007; 34-35:35-38. [en ligne] Disponible sur: http://www.enssib.fr/ bibliotheque-numerique/documents/59316-34-35-sciences-ettechniques.pdf.

9. Espaces Rencontres Information (ERI), Lieux d'accompagnement et/ou d'info. Paris: Institut National Du Cancer. [Site Internet: consulté le 17 octobre 2017]. Disponible sur: http://www.e-cancer.fr/Patients-et-proches/Ressources-utiles/ Lieux-d-accompagnement-et-ou-d-info/Espaces-RencontresInformation-ERI. [Site Internet: consulté le 17 octobre 2017].

10. Fédération Hospitalière de France. Les maisons des usagers. Disponible sur: https://www.hopital.fr/Droits-demarches/Vosdroits/La-representation-des-usagers-et-le-respect-des-droits/ Les-maisons-des-usagers.

11. International Federation of Library Associations and Institutions. Guide à des bibliothèques desservant des patients hospitalisés, des personnes âgées et handicapées dans des institutions de long séjour [en ligne]. IFLA Professional Reports. 2004; 84: 56p [consulté le 23 septembre 2016]. Disponible sur: http://www.ifla.org/files/assets/hq/publica tions/professional-report/83.pdf.

12. Texier $\mathrm{B}$, Archimag. Bibliothèques à l'hôpital: une présence inégale auprès des malades [en ligne; accès restreint]. Mise en ligne le 09/07/2014 [consulté le 23/09/2016]. Disponible sur: http://www.archimag.com/bibliotheque-edition/2014/07/09/ bibliotheques-hopital-presence-inegale-malades.

13. Haute Autorité de Santé. Éducation Thérapeutique du Patient: définition, finalités et organisation [en ligne], Saint-Denis La Plaine: Haute Autorité de Santé; 2007. Disponible sur : https:// www.has-sante.fr/portail/upload/docs/application/pdf/etp_definition_finalites___recommandations_juin_2007.pdf.

14. Organisation de coopération et de développement économiques. Country Note: France [en ligne]. 2013. Disponible sur: http:// www.oecd.org/skills/piaac/Country\%20note $\% 20-\% 20$ France $\%$ 20(FR).pdf. [consulté le 19 octobre 2017]

Citation de l'article : de Andrade V, Margat A, Verheye J-C, Gagnayre R. Information, éducation du patient et littératie en santé : un rôle à jouer pour les bibliothécaires/documentalistes en France? Educ Ther Patient/Ther Patient Educ 2018;10:10502. 